\title{
Assessment of Musculoskeletal Ultrasound Guided Injection of The New Regenerative Medicine Techniques (Neural Prolotherapy and Platelet Rich Plasma) Impact versus Well Established Techniques (Steroid Injection) in Cases of Carpal Tunnel Syndrome Secondary to Rheumatoid Arthritis
} Ahmed A. Abdel-Aziz ${ }^{1}$, Hegazy M. Al-Tamimy ${ }^{2}$, Ahmed M. Fahmy ${ }^{3}$ Mahmoud S. Abd Allah ${ }^{4}$

1,2,3,4 Department of Rheumatology and Rehabilitation, Faculty of Medicine, Al-Azhar University, Cairo, Egypt. Corresponding Author: Mahmoud S. Abd Allah; Mobile: 011539.2532; Email: Samymahmoud662@yahoo.com

\begin{abstract}
Background: Rheumatoid arthritis is a systemic rheumatic disease characterized by symmetrical, often erosive and deforming poly-arthritis with extra-articular manifestations in 10-20\% of patients, especially those with high titers of rheumatoid factor. Extra articular pathology includes bursitis, tendonitis and neuritis, which results from entrapment, nerve ischemia due to vasculitis or drugs used to treat this condition. Carpal tunnel syndrome is the most common compression neuropathy associated with rheumatoid arthritis.

Aim of the Work: To evaluate the efficacy of Neural Prolotherapy and Platelet Rich Plasma in treatment of carpal tunnel syndrome secondary to rheumatoid arthritis.

Patients and Methods: Ninety patients with Rheumatoid Arthritis (RA) that were all fulfilling the 2016 ACR/EULAR classification criteria for RA. All were over the age of sixteen years at time of diagnosis, complaining of burning pain or paresthesia in the median nerve distribution of the hand. They were recruited from Rheumatology and Rehabilitation Department at Al-Hussein and Sayed Galal University Hospitals during the period from December 2018 to July 2019.

Results: Neural Prolotherapy and Platelet Rich Plasma (PRP) have improved all measured parameters like visual analogue scale (VAS), nerve conduction studies and neuromuscular ultrasonography parameters in carpal tunnel syndrome secondary to rheumatoid arthritis.

Conclusion: Neural Prolotherapy and Platelet Rich Plasma proved to be effective treatments of carpal tunnel syndrome secondary to rheumatoid arthritis.
\end{abstract}

Keywords: Rheumatoid arthritis, Carpal tunnel syndrome, Steroid, Neural prolotherapy and Platelet Rich Plasma.

\section{INTRODUCTION}

Rheumatoid arthritis is a systemic rheumatic disease characterized by symmetrical, often erosive and deforming poly-arthritis. Extra-articular manifestations occur in 10-20\% of patients, especially those with high titers of rheumatoid factor. Extra articular pathology includes bursitis, tendonitis, neuritis, and vasculitis (1). Rheumatoid neuropathy could result from nerve entrapment, drug toxicity, vasculitis, amyloidosis and autoimmune phenomenon (2). Carpal tunnel syndrome is the most common compression neuropathy associated with rheumatoid arthritis. Pain and/or paresthesias at night as well as weakness, loss of dexterity and thenar atrophy can occur ${ }^{(3)}$. Symptoms of neuropathy may be overlooked or overestimated in the presence of severe joint disease, restriction, pain and deformities. Careful examination is thus warranted while evaluating such patients ${ }^{(4)}$. Clinical neuropathy may present with a wide variety of symptoms, such as pain, paresthesia, and muscle weakness ${ }^{(5)}$. Most compressive neuropathies can be diagnosed clinically, electromyography and nerve conduction studies can confirm the diagnosis and usually are required before surgery (6). The neuromuscular ultrasound can detect pathologies that may predispose or lead to entrapment neuropathy ${ }^{(7)}$. Over the past years, high-resolution ultrasonography has been proposed as a useful tool for the diagnosis of carpal tunnel syndrome (8). Glucocorticoids not only have anti-inflammatory effects, but they can also play a role in the recovery of myelination after a peripheral nerve injury ${ }^{(9)}$. Injection of glucocorticoids into the region of the carpal tunnel is intended to reduce tissue inflammation and aid recovery ${ }^{\left({ }^{10}\right)} .5 \%$ dextrose (D5W) has been commonly used in peripheral entrapment neuropathy, because D5W possesses osmolarity similar to that of normal saline and no harmful effects have been reported from animal and human studies ${ }^{\mathbf{1 1 1}}$.

It is hypothesized that D5W could decrease neurogenic inflammation ${ }^{(12)}$. Perineural injection of PRP proved to be significantly more effective than platelet poor plasma (PPP) in visual analogue scale (VAS) and two-point discrimination test. This result is in favor of the positive effect of PRP in nerve regeneration ${ }^{(13)}$. Platelet rich plasma may be effective in controlling median nerve injury ${ }^{(\mathbf{1 4})}$.

\section{AIM OF THE WORK}

The aim of the work is to evaluate the efficacy of the new regenerative medicine techniques (Neural Prolotherapy and Platelet Rich Plasma) in treatment of carpal tunnel syndrome secondary to rheumatoid arthritis. 


\section{PATIENTS AND METHODS}

Ethical approval:

The ethical approval was obtained from the

Hospital Ethical Research Committee and each patient included in the study signed an informed consent. Ninety Egyptian patients with Rheumatoid Arthritis (RA), all fulfilled the 2010 ACR/EULAR classification criteria for RA and/or the 2016 ACR/EULAR classification criteria for RA. All were over the age of sixteen years at time of diagnosis. Mild to moderate CTS-diagnosed according to American Association of the Electrodiagnostic Medicine (AAEM) criteria. They were recruited from Rheumatology and Rehabilitation Outpatient Clinic at Al-Hussein and Sayed Galal University Hospitals during the period from December 2018 to July 2019.

Inclusion criteria (group A): Ninety patients complaining of burning pain or paresthesia in the median nerve distribution of the hand (the thumb, the index finger, the middle finger, and the radial side of the ring finger) with positive Phalen and/or Tinel test and Electrophysiological study confirming the diagnosis of CTS.

Exclusion criteria: Hand/wrist trauma, fractures and surgery, congenital or post-traumatic hand deformity, patients that well known to have neurological disease with peripheral neuropathy e.g.(guillain barre syndrome), systemic diseases other than RA e.g. (diabetes mellitus, hypothyroidism), pregnancy, current warfarin use, previous steroid injection for CTS, trauma, infection and neoplasm at injectionsite.

Control group (group B): Thirty healthy individuals, age and sex matched, were used as a control group.

Patients group (group A): Ninety patients had been sub-classified randomly into three equal groups: group (1); thirty patients have received a single dose of $40 \mathrm{mg}$ triamcinolone acetonide \{Epirelefan manufactured by EIPICO that locally injected into the affected carpal tunnel, group (2); thirty patients have received 2-3 $\mathrm{ml}$ 5\% Dextrose buffered by Sodium Bicarbonate $8.4 \%$ injected into the affected carpal tunnel at 4-6 sessions 10 days apart and group (3); thirty patients have received 1-2 ml Platelet Rich Plasma injected into the affected carpal tunnel at 2 sessions 2 weeks apart.

All patients were subjected to:

1) Clinical evaluation: included full history taking, clinical examination, musculoskeletal examination, neurological examination, disease activity score (DAS) 28 and special tests for CTS (Phalen's test and Tinel's test).

2) Laboratory evaluation in the form of: CBC, ESR, CRP, $\mathrm{RF}$, anti-CCP, fasting and 2 hours postprandial blood sugar and TSH.
3) Electrophysiological study at $0 \& 3$ months after treatment for: sensory conduction studies (SCS), motor conduction studies (MCS) and for response of median nerve in the affected side.

4) Musculoskeletal Ultrasonography at $0 \& 3$ months after treatment for: cross sectional srea of the median nerve at the proximal inlet of carpal tunnel, vascularity, echogenicity, mobility and flattening Ratio of the median nerve.

PRP preparation: The whole blood of $10 \mathrm{ml}$ was taken from each patient. The blood is collected on citrated tube with the mixing rate was 9:1 in volume and mixing by inversion. The tubes were centrifuged (first centrifugation). The rotation speed and time was $3000 \mathrm{rpm}$ for $3 \mathrm{~min}$, which was the minimum for separating red blood cells (RBCs) from plasma. The tubes were then taken out from the centrifuge and arranged on a holder and the plasma was collected by syringes and transferred to another sterile tube without anticoagulant and was centrifuged. The second centrifugation was performed on plasma tube at 4000 rpm for $15 \mathrm{~min}$, which is the fastest speed of the machine and considered to be the realistic time as a daily practice. The supernatant platelet poor plasma (PPP) was removed leaving $2 \mathrm{ml}$ of PRP on sediment (platelet pellet), and suspend the platelet pellets by gently shaking the tube.

Technique of Injection: Applying antiseptic to skin (Betadine \& Alcohol) to provide complete aseptic condition, 27 gauge 1.25 inch (or 25 gauge 1.5 inch) was used and real time ultrasound-guided injection around the median nerve was done.

\section{Statistical analysis}

Recorded data were analyzed using the statistical package for social sciences, version 20.0 (SPSS Inc., Chicago, Illinois, USA). Quantitative data were expressed as mean \pm standard deviation (SD). Qualitative data were expressed as frequency and percentage. The following tests were done: Independent-samples t- test of significance was used when comparing between two means. A one-way analysis of variance (ANOVA) when comparing between more than two means, Post Hoc test. Least Significant Difference (LSD) was used for multiple comparisons between different variables. Chi-square $\left(\mathrm{x}^{2}\right)$ test of significance was used in order to compare proportions between qualitative parameters. The confidence interval was set to $95 \%$ and the margin of error accepted was set to 5\%. So, the p-value was considered significant as the following: Probability (P-value) Pvalue $\leq 0.05$ was considered significant, $P$-value $\leq 0.001$ was considered as highly significant, $\mathrm{P}$-value $>0.05$ was considered insignificant. 


\section{RESULTS}

Table (1): Comparison between groups according to demographic data.

\begin{tabular}{|c|c|c|c|c|c|}
\hline $\begin{array}{l}\text { Demographic } \\
\text { data }\end{array}$ & $\begin{array}{l}\text { Group Ia: } \\
\text { Steroid }(n=30)\end{array}$ & $\begin{array}{l}\text { Group Ib: } \\
\text { Prolotherapy }(n=30)\end{array}$ & $\begin{array}{l}\text { Group Ic: } \\
\operatorname{PRP}(n=30)\end{array}$ & F/x2\# & p- value \\
\hline $\begin{array}{l}\text { Gender } \\
\text { Male } \\
\text { Female }\end{array}$ & $\begin{array}{l}3(10.0 \%) \\
27(90.0 \%)\end{array}$ & $\begin{array}{l}6(20.0 \%) \\
24(80.0 \%)\end{array}$ & $\begin{array}{l}9(30.0 \%) \\
21(70.0 \%)\end{array}$ & 3.927 & 0.218 \\
\hline $\begin{array}{l}\text { Age (years) } \\
\text { Mean } \pm S D \\
\text { Range }\end{array}$ & $\begin{array}{c}50.00 \pm 11.88 \\
24-68\end{array}$ & $\begin{array}{l}47.10 \pm 15.10 \\
25-65\end{array}$ & $\begin{array}{c}48.50 \pm 10.11 \\
29-58\end{array}$ & 1.233 & 0.210 \\
\hline
\end{tabular}

F-One Way Analysis of Variance; $\# x^{2}$ : Chi-square test;

p-value >0.05 NS; *p-value <0.05 $\mathrm{S}$;**p-value <0.001 HS

This table showed no statistically significant difference between groups according to demographic data.

Table (2): Comparison between groups according to duration of Rheumatoid Arthritis (years).

\begin{tabular}{|c|c|c|c|c|c|}
\hline Duration (years) & $\begin{array}{l}\text { Group Ia: } \\
\text { Steroid } \\
(n=30)\end{array}$ & $\begin{array}{l}\text { Group Ib: } \\
\text { Prolotherapy } \\
(n=30)\end{array}$ & $\begin{array}{l}\text { Group Ic: } \\
\operatorname{PRP}(n=30)\end{array}$ & ANOVA & $\begin{array}{l}\text { p- } \\
\text { value }\end{array}$ \\
\hline Mean \pm SD & $9.10 \pm 6.83$ & $8.54 \pm 5.47$ & $8.20 \pm 4.26$ & \multirow[b]{2}{*}{4.731} & \multirow[b]{2}{*}{0.193} \\
\hline Range & $1-22$ & $2-16$ & $2-13$ & & \\
\hline
\end{tabular}

F-One Way Analysis of Variance; $p$-value >0.05 NS;

This table showed no statistically significant difference between groups according to duration of RA (years).

Table (3): Comparison between groups according to DAS 28 score.

\begin{tabular}{|l|l|l|l|l|l|}
\hline DAS score & $\begin{array}{l}\text { Group Ia: } \\
\text { Steroid }(\boldsymbol{n}=\mathbf{3 0})\end{array}$ & $\begin{array}{l}\text { Group Ib: } \\
\text { Prolotherapy }(\boldsymbol{n}=\mathbf{3 0})\end{array}$ & $\begin{array}{l}\text { Group Ic: } \\
\text { PRP }(\boldsymbol{n}=\mathbf{3 0})\end{array}$ & ANOVA & p- value \\
\hline $\begin{array}{l}\text { Before treatment } \\
\text { Mean } \pm \text { SD }\end{array}$ & $2.61 \pm 0.41$ & $2.86 \pm 0.53$ & $2.90 \pm 0.75$ & 2.167 & 0.121 \\
Range & $1.96-3.4$ & $2.35-4.1$ & $2.19-4.9$ & & \\
\hline $\begin{array}{l}\text { After 3 months } \\
\text { Mean } \pm \text { SD }\end{array}$ & $2.60 \pm 0.41$ & $\begin{array}{l}2.86 \pm 0.48 \mathrm{a} \\
\text { Range }\end{array}$ & $\begin{array}{l}2.88 \pm 0.66 \mathrm{a} \\
2.2-4.58\end{array}$ & 1.855 & 0.053 \\
\hline
\end{tabular}

F-One Way Analysis of Variance;

Post HOC: : Significant difference with group

steroid; $p$-value $>0.05 \mathrm{NS} ;{ }^{*}$ p-value $<0.05 \mathrm{~S}$

This table showed no statistically significant difference between groups according to DAS 28 score.

Table (4): Comparison between groups according to Visual Analogue Scale (VAS).

\begin{tabular}{|c|c|c|c|c|c|}
\hline VAS score & Group Ia: & Group Ib: & Group Ic: & ANOVA & p-value \\
\hline & Steroid $(n=30)$ & $\begin{array}{l}\text { Prolotherapy } \\
(\mathbf{n}=\mathbf{3 0})\end{array}$ & PRP $(n=30)$ & & \\
\hline \multicolumn{4}{|c|}{ Before treatment } & \multirow{3}{*}{1.995} & \multirow{3}{*}{0.142} \\
\hline Mean \pm SD & $7.85 \pm 1.02$ & $7.80 \pm 0.76$ & $7.40 \pm 1.06$ & & \\
\hline Range & 6-9 & $7-9$ & 6-9 & & \\
\hline \multicolumn{4}{|c|}{ After 3 months } & \multirow{3}{*}{3.455} & \multirow{3}{*}{$0.036^{*}$} \\
\hline Mean \pm SD & $2.10 \pm 0.84$ & $1.70 \pm 0.65 a$ & $1.50 \pm 1.14 a b$ & & \\
\hline Range & $1-3$ & $1-3$ & $0-3$ & & \\
\hline $\begin{array}{l}\text { Mean } \\
\text { Difference }\end{array}$ & $-5.75 \pm 1.09$ & $-6.10 \pm 1.16$ & $-5.90 \pm 1.12$ & 1.184 & 0.421 \\
\hline
\end{tabular}

F-One Way Analysis of Variance;

\section{Post HOC:}

a: Significant difference with group steroid;

b: Significant difference with group prolotherapy; p-value >0.05 NS; *p-value <0.05 S;

This table showed statistically significant difference between groups according to VAS after 3 months, while mean difference between groups insignificant. 
Table (5): Comparison between groups according to F-wave.

\begin{tabular}{|l|l|l|l|l|l|}
\hline Fwave & $\begin{array}{l}\text { Group Ia: } \\
\text { Steroid } \\
(\boldsymbol{n}=\mathbf{3 0})\end{array}$ & $\begin{array}{l}\text { Group Ib: } \\
\text { Prolotherapy } \\
(\boldsymbol{n}=\mathbf{3 0})\end{array}$ & $\begin{array}{l}\text { Group Ic: } \\
\text { PRP }(\boldsymbol{n = 3 0})\end{array}$ & ANOVA & p-value \\
\hline Mean \pm SD & $26.36 \pm 1.41$ & $26.85 \pm 1.88$ & $26.32 \pm 1.54$ & 1.533 & 0.482 \\
\hline Range & $24-29$ & $25-29$ & $23.1-28.4$ & & \\
\hline
\end{tabular}

F-One Way Analysis of Variance; $p$-value $>0.05$ NS

This table showed no statistically significant difference between groups according to F-wave.

Table (6): Comparison between groups according to nerve conduction study (before treatment).

\begin{tabular}{|c|c|c|c|c|c|}
\hline $\begin{array}{l}\text { Nerve conduction study } \\
\text { (Before treatment) }\end{array}$ & $\begin{array}{l}\text { Group Ia: } \\
\text { Steroid } \\
(n=30)\end{array}$ & $\begin{array}{l}\text { Group Ib: } \\
\text { Prolotherapy } \\
(n=30) \\
\end{array}$ & $\begin{array}{l}\text { Group Ic: } \\
\operatorname{PRP}(n=30)\end{array}$ & ANOVA & p-value \\
\hline $\begin{array}{l}\text { Distal Motor Latency } \\
\text { Mean } \pm \text { SD } \\
\text { Range } \\
\end{array}$ & $\begin{array}{l}4.66 \pm 0.51 \\
4.02-5.7 \\
\end{array}$ & $\begin{array}{l}4.43 \pm 1.12 \\
2.14-6.46 \\
\end{array}$ & $\begin{array}{l}4.42 \pm 0.78 \\
3.46-6.28 \\
\end{array}$ & 1.203 & 0.167 \\
\hline $\begin{array}{l}\text { Motor Amplitude } \\
\text { Mean } \pm \text { SD } \\
\text { Range }\end{array}$ & $\begin{array}{l}4.85 \pm 1.07 \\
3.7-7.04\end{array}$ & $\begin{array}{l}5.37 \pm 1.33 \\
3.62-7.48\end{array}$ & $\begin{array}{l}5.06 \pm 1.82 \\
3.11-8.56\end{array}$ & 2.305 & 0.095 \\
\hline $\begin{array}{l}\text { Motor Conduction } \\
\text { Velocity } \\
\text { Mean } \pm \text { SD } \\
\text { Range } \\
\end{array}$ & $\begin{array}{l}46.75 \pm 6.95 \\
39-64\end{array}$ & $\begin{array}{l}45.99 \pm 3.71 \\
42-53.8\end{array}$ & $\begin{array}{l}52.73 \pm 6.82 \mathrm{ab} \\
43.8-67.3\end{array}$ & 10.800 & $<0.001 * *$ \\
\hline $\begin{array}{l}\text { Distal Sensory Latency } \\
\text { Mean } \pm \text { SD } \\
\text { Range }\end{array}$ & $\begin{array}{l}4.34 \pm 1.58 \\
3.42-8.86\end{array}$ & $\begin{array}{l}3.74 \pm 0.20 \mathrm{a} \\
3.4-4.03\end{array}$ & $\begin{array}{l}3.68 \pm 0.33 \mathrm{a} \\
3.24-4.3\end{array}$ & 3.799 & $0.010^{*}$ \\
\hline $\begin{array}{l}\text { Sensory Amplitude } \\
\text { Mean } \pm \text { SD } \\
\text { Range } \\
\end{array}$ & $\begin{array}{l}15.59 \pm 2.34 \\
12.5-18.63\end{array}$ & $\begin{array}{l}17.57 \pm 1.51 \\
13.5-18.7\end{array}$ & $\begin{array}{l}17.29 \pm 0.87 \\
15.7-18.6\end{array}$ & 0.449 & 0.153 \\
\hline $\begin{array}{l}\text { Sensory Conduction } \\
\text { Velocity } \\
\text { Mean } \pm \text { SD } \\
\text { Range }\end{array}$ & $\begin{array}{c}42.03 \pm 1.51 \\
39-44.1\end{array}$ & $\begin{array}{r}42.08 \pm 1.75 \\
39-44\end{array}$ & $\begin{array}{r}43.58 \pm 1.35 \\
41.4-47\end{array}$ & 0.615 & 0.270 \\
\hline
\end{tabular}

F-One Way Analysis of Variance;

Post HOC:

a: Significant difference with group steroid;

b: Significant difference with group

prolotherapy; c: Significant difference with

group PRP

p-value >0.05 NS; *p-value <0.05 S; **p-value <0.001 HS

This table showed statistically significant difference between groups according to nerve conduction study

(Motor Conduction Velocity \& Distal Sensory Latency) before treatment.

Table (7): Comparison between before treatment and 3 months after local injection of steroid according to nerve conduction study.

\begin{tabular}{|l|l|l|l|l|l|}
\hline $\begin{array}{l}\text { Nerve conduction } \\
\text { study }\end{array}$ & $\begin{array}{l}\text { Before } \\
\text { Treatment }\end{array}$ & $\begin{array}{l}\text { After } \\
\text { 3months }\end{array}$ & Mean Diff. & t-test & p-value \\
\hline Distal Motor Latency & $4.66 \pm 0.51$ & $3.67 \pm 0.18$ & -0.99 & 12.722 & $<0.001^{* *}$ \\
\hline Motor Amplitude & $4.85 \pm 1.07$ & $6.16 \pm 1.01$ & 1.31 & -4.390 & $<0.001^{* *}$ \\
\hline Motor ConductionVelocity & $46.75 \pm 6.95$ & $53.41 \pm 7.07$ & 6.66 & -4.696 & $<0.001^{* *}$ \\
\hline DSL & $4.34 \pm 1.58$ & $2.47 \pm 0.15$ & -1.87 & 6.781 & $<0.001^{* *}$ \\
\hline SA & $15.59 \pm 2.34$ & $25.63 \pm 1.91$ & 10.04 & -14.378 & $<0.001^{* *}$ \\
\hline SNCV & $42.03 \pm 1.51$ & $54.79 \pm 7.26$ & 12.76 & -9.219 & $<0.001^{* *}$ \\
\hline
\end{tabular}

$t$-Paired Sample t-test; **p-value <0.001 HS

This table showed statistically significant difference between before treatment and 3 months after local injection of steroid according to nerve conduction study. 
Table (8): Comparison between before treatment and 3 months after local injection of Neural Prolotherapy according to nerve conduction study.

\begin{tabular}{|l|l|l|l|l|l|}
\hline Nerve conduction study & $\begin{array}{l}\text { Before } \\
\text { Treatment }\end{array}$ & After 3months & $\begin{array}{l}\text { Mean } \\
\text { Diff. }\end{array}$ & t-test & p-value \\
\hline Distal Motor Latency & $4.43 \pm 1.12$ & $3.57 \pm 0.38$ & -0.86 & 3.738 & $<0.001^{* *}$ \\
\hline Motor Amplitude & $5.37 \pm 1.33$ & $5.91 \pm 0.97$ & 0.54 & -1.942 & $0.045^{*}$ \\
\hline Motor Conduction Velocity & $45.99 \pm 3.71$ & $48.61 \pm 4.57$ & 2.62 & -2.123 & $0.042^{*}$ \\
\hline DSL & $3.74 \pm 0.20$ & $2.63 \pm 0.55$ & -1.11 & 11.696 & $<0.001^{* *}$ \\
\hline SA & $17.57 \pm 1.51$ & $26.73 \pm 1.06$ & 9.16 & -22.483 & $<0.001^{* *}$ \\
\hline SNCV & $42.08 \pm 1.75$ & $53.61 \pm 4.69$ & 11.53 & -11.348 & $<0.001^{* *}$ \\
\hline
\end{tabular}

t-Paired Sample t-test

p-value >0.05 NS; *p-value <0.05 S;**p-value <0.001 HS

This table showed statistically significant difference between before treatment and 3 months after local injection of Neural Prolotherapy according to nerve conduction study.

Table (9): Comparison between before treatment and and 3 months after local injection of PRP according to nerve conduction study.

\begin{tabular}{|l|l|l|l|l|l|}
\hline Nerve conduction study & $\begin{array}{l}\text { Before } \\
\text { Treatment }\end{array}$ & After 3months & $\begin{array}{l}\text { Mean } \\
\text { Diff. }\end{array}$ & t-test & p-value \\
\hline Distal Motor Latency & $4.42 \pm 0.78$ & $3.58 \pm 0.18$ & -0.84 & 6.215 & $<0.001^{* *}$ \\
\hline Motor Amplitude & $5.06 \pm 1.82$ & $6.02 \pm 0.83$ & 0.96 & -2.232 & $0.034^{*}$ \\
\hline Motor Velocity & $52.73 \pm 6.82$ & $57.67 \pm 3.93$ & 4.94 & 5.481 & $<0.001^{* *}$ \\
\hline DSL & $3.68 \pm 0.33$ & $2.57 \pm 0.21$ & -1.11 & 12.787 & $<0.001^{* *}$ \\
\hline SA & $17.29 \pm 0.87$ & $26.58 \pm 2.36$ & 9.29 & -18.529 & $<0.001^{* *}$ \\
\hline SNCV & $43.58 \pm 1.35$ & $53.30 \pm 6.32$ & 9.72 & -7.459 & $<0.001^{* *}$ \\
\hline
\end{tabular}

t-Paired Sample t-test

p-value $>0.05 \mathrm{NS} ;{ }^{*}$-value $<0.05 \mathrm{~S}$; **p-value $<0.001 \mathrm{HS}$

This table showed statistically significant difference between before treatment and 3 months after local injection of PRP according to nerve conduction study.

Table (10): Comparison between groups according to mean difference before and 3 months after local injection of nerve conduction study.

\begin{tabular}{|l|l|l|l|l|l|}
\hline $\begin{array}{l}\text { Mean difference before and } \\
\text { after treatment of } \\
\text { nerve conduction study }\end{array}$ & $\begin{array}{l}\text { Group Ia: } \\
\text { Steroid } \\
(\boldsymbol{n}=\mathbf{3 0})\end{array}$ & $\begin{array}{l}\text { Group Ib: } \\
\text { Prolotherapy } \\
(\boldsymbol{n}=\mathbf{3 0})\end{array}$ & $\begin{array}{l}\text { Group Ic: } \\
\text { PRP } \\
(\boldsymbol{n = 3 0 )}\end{array}$ & ANOVA & p-value \\
\hline Distal Motor Latency & $-0.99 \pm 0.33$ & $-0.86 \pm 0.28 \mathrm{a}$ & $-0.84 \pm 0.28 \mathrm{a}$ & 1.482 & $0.049^{*}$ \\
\hline Motor Amplitude & $1.31 \pm 0.43$ & $0.54 \pm 0.18 \mathrm{a}$ & $0.96 \pm 0.32 \mathrm{ab}$ & 6.461 & $<0.001^{* *}$ \\
\hline Motor Conduction Velocity & $6.66 \pm 2.20$ & $2.62 \pm 0.86 \mathrm{a}$ & $4.94 \pm 1.01 \mathrm{ab}$ & 5.197 & $<0.001^{* *}$ \\
\hline Distal Sensory Latency & $-1.87 \pm 0.62$ & $-1.11 \pm 0.37 \mathrm{a}$ & $-1.11 \pm 0.37 \mathrm{a}$ & 3.971 & $0.014^{*}$ \\
\hline Sensory Amplitude & $10.04 \pm 3.31$ & $9.16 \pm 3.02 \mathrm{a}$ & $9.29 \pm 3.07 \mathrm{a}$ & 2.163 & $0.017^{*}$ \\
\hline Sensory Conduction Velocity & $12.76 \pm 4.21$ & $11.53 \pm 3.80 \mathrm{a}$ & $9.72 \pm 3.21 \mathrm{ab}$ & 4.663 & $0.008^{*}$ \\
\hline
\end{tabular}

F-One Way Analysis of Variance;

\section{Post HOC:}

a: Significant difference with group steroid;

$b$ : Significant difference with group

prolotherapy; c: Significant difference with

group PRP

*p-value $<0.05 \mathrm{~S}$; **p-value $<0.001 \mathrm{HS}$

This table showed statistically significant difference between groups according to mean difference before and 3 months after local injection of nerve conduction study. 
Table (11): Comparison between groups according to ultrasound before treatment.

\begin{tabular}{|l|l|l|l|l|l|}
\hline $\begin{array}{l}\text { Ultrasound } \\
\text { (Before } \\
\text { treatment) }\end{array}$ & $\begin{array}{l}\text { Group Ia: } \\
\text { Steroid } \\
(\boldsymbol{n = 3 0 )}\end{array}$ & $\begin{array}{l}\text { Group Ib: } \\
\text { Prolotherapy } \\
(\boldsymbol{n}=\mathbf{3 0})\end{array}$ & $\begin{array}{l}\text { Group Ic: } \\
\text { PRP }(\boldsymbol{n}=\mathbf{3 0})\end{array}$ & F/x2\# & p-value \\
\hline $\begin{array}{l}\text { CSA } \\
\text { Mean } \pm \text { SD } \\
\text { Range }\end{array}$ & $\begin{array}{l}14.28 \pm 1.49 \\
12.3-16.4\end{array}$ & $\begin{array}{l}13.33 \pm 0.85 \\
12.3-14.8\end{array}$ & $\begin{array}{l}13.47 \pm 1.16 \\
12-15.7\end{array}$ & 2.015 & 0.208 \\
\hline $\begin{array}{l}\text { Vascularity } \\
\text { Normal }\end{array}$ & $18(60.0 \%)$ & $18(60.0 \%)$ & $\begin{array}{l}12(40.0 \%) \\
12(40.0 \%)\end{array}$ & 4.146 & 0.451 \\
$\begin{array}{l}\text { Minimal } \\
\text { Moderate }\end{array}$ & $12(40.0 \%)$ & $\begin{array}{l}12(40.0 \%) \\
0(0.0 \%)\end{array}$ & $6(20.0 \%)$ & & \\
\hline $\begin{array}{l}\text { Mobility } \\
\text { Mobile }\end{array}$ & $30(100.0 \%)$ & $30(100.0 \%)$ & $30(100.0 \%)$ & $0.000 \#$ & 1.000 \\
\hline $\begin{array}{l}\text { Flattening Ratio } \\
\text { Mean } \pm \text { SD } \\
\text { Range }\end{array}$ & $3.04 \pm 0.34$ & $3.04 \pm 0.43$ & $2.99 \pm 0.46$ & 0.640 & 0.262 \\
\hline
\end{tabular}

F-One Way Analysis of Variance; $\# x^{2}$ : Chi-square test; $p$ -

value >0.05 NS;

This table showed statistically significant difference between groups according to CSA, vascularity and FR.

Table (12): Comparison between before treatment and 3 months after local injection of Steroid according to Ultrasound

\begin{tabular}{|c|c|c|c|c|c|}
\hline Ultrasound & \begin{tabular}{|l|} 
Before \\
Treatment
\end{tabular} & \begin{tabular}{|l|} 
After \\
3months
\end{tabular} & $\begin{array}{l}\text { Mean } \\
\text { Diff. }\end{array}$ & $t / x 2 \#$ & p-value \\
\hline CSA & $14.28 \pm 1.49$ & $12.30 \pm 1.26$ & -1.98 & 30.086 & $<0.001 * *$ \\
\hline $\begin{array}{l}\text { Vascularity } \\
\text { Normal } \\
\text { Minimal } \\
\text { Moderate }\end{array}$ & $\begin{array}{l}18(60.0 \%) \\
12(40.0 \%) \\
0(0.0 \%)\end{array}$ & $\begin{array}{l}30(100.0 \%) \\
0(0 \%) \\
0(0 \%)\end{array}$ & $\begin{array}{l}-- \\
-- \\
--\end{array}$ & 12.604\# & $0.004 *$ \\
\hline FR & $3.04 \pm 0.34$ & $2.56 \pm 0.31$ & -0.48 & 45.524 & $<0.001 * *$ \\
\hline
\end{tabular}

t-Paired Sample t-test; $\# x^{2}$ : Chi-square test;

*p-value $<0.05 \mathrm{~S} ; * *$-value $<0.001 \mathrm{HS}$

This table showed statistically significant difference between before treatment and 3 months after local injection of Steroid according to Ultrasound.

Table (13): Comparison between before treatment and 3 months after local injection of Neural Prolotherapy according to Ultrasound

\begin{tabular}{|l|l|l|l|l|l|}
\hline Ultrasound & $\begin{array}{l}\text { Before } \\
\text { Treatment }\end{array}$ & $\begin{array}{l}\text { After } \\
\text { 3months }\end{array}$ & Mean Diff. & t/x2\# & p-value \\
\hline CSA & $13.33 \pm 0.85$ & $11.33 \pm 0.65$ & -2 & 33.081 & $<0.001^{* *}$ \\
\hline Vascularity & $18(60.0 \%)$ & $30(100.0 \%)$ & -- & & \\
Normal & $12(40.0 \%)$ & $0(0 \%)$ & -- & $12.604 \#$ & $0.004^{*}$ \\
Minimal & $0(0.0 \%)$ & $0(0 \%)$ & -- & & \\
Moderate & $3.04 \pm 0.43$ & $2.65 \pm 0.39$ & -0.39 & 22.822 & $<0.001^{* *}$ \\
\hline FR
\end{tabular}

t-Paired Sample t-test; $\# x^{2}$ : Chi-square test;

${ }^{*}$ p-value $<0.05 \mathrm{~S}$;**p-value $<0.001 \mathrm{HS}$

This table showed statistically significant difference between before treatment and 3 months after local injection of Neural Prolotherapy according to Ultrasound. 
Table (14): Comparison between before treatment and 3 months after local injection of PRP according to Ultrasound.

\begin{tabular}{|l|l|l|l|l|c|}
\hline Ultrasound & $\begin{array}{l}\text { Before } \\
\text { Treatment }\end{array}$ & After 3months & Mean Diff. & t/x2\# & p-value \\
\hline CSA & $13.47 \pm 1.16$ & $11.58 \pm 1.05$ & -1.89 & 29.517 & $<0.001^{* *}$ \\
\hline Vascularity & $12(40.0 \%)$ & $30(100.0 \%)$ & -- & & \\
Normal & $12(40.0 \%)$ & $0(0 \%)$ & -- & $25.714 \#$ & $<0.001^{* *}$ \\
Minimal & $6(20.0 \%)$ & $0(0 \%)$ & -- & & \\
Moderate & $2.99 \pm 0.46$ & $2.53 \pm 0.44$ & -0.46 & 45.213 & $<0.001^{* *}$ \\
\hline FR &
\end{tabular}

t-Paired Sample t-test; $\# x^{2}$ : Chi-square test; **p-value <0.001 HS

This table showed statistically significant difference between before treatment and 3 months after local injection of PRP according to Ultrasound.

Table (15): Comparison between groups according to mean difference before and 3 months after local injection of ultrasound.

\begin{tabular}{|l|l|l|l|l|l|}
\hline $\begin{array}{l}\text { Mean difference before } \\
\text { and after treatment of } \\
\text { Ultrasound }\end{array}$ & $\begin{array}{l}\text { Group Ia: } \\
\text { Steroid }(\boldsymbol{n}=\mathbf{3 0})\end{array}$ & $\begin{array}{l}\text { Group Ib: } \\
\text { Prolotherapy } \\
(\boldsymbol{n}=\mathbf{3 0})\end{array}$ & $\begin{array}{l}\text { Group Ic: } \\
\text { PRP }(\boldsymbol{n = 3 0})\end{array}$ & ANOVA & p-value \\
\hline CSA & $-1.98 \pm 0.65$ & $-2.00 \pm 0.66$ & $-1.89 \pm 0.62 \mathrm{ab}$ & 2.714 & $0.023^{*}$ \\
\hline FR & $-0.48 \pm 0.16$ & $-0.39 \pm 0.13 \mathrm{a}$ & $-0.46 \pm 0.15 \mathrm{~b}$ & 4.669 & $0.006^{*}$ \\
\hline
\end{tabular}

F-One Way Analysis of Variance;

\section{Post HOC:}

a: Significant difference with group steroid;

b: Significant difference with group prolotherapy;

*p-value $<0.05 \mathrm{~S}$

This table showed statistically significant difference between groups according to mean difference before and 3 months after local injection of ultrasound.

Table (16): Comparison between patients and control according to demographic data.

\begin{tabular}{|c|c|c|c|c|c|}
\hline & Demographic data & Patients $(n=90)$ & Control $(n=30)$ & $t / \mathbf{x} 2 \#$ & p-value \\
\hline Age (years) & $\begin{array}{l}\text { Mean } \pm \text { SD } \\
\text { Range }\end{array}$ & $\begin{array}{l}46.20 \pm 12.89 \\
24-68\end{array}$ & $\begin{array}{l}47.50 \pm 10.43 \\
24-62\end{array}$ & 1.195 & 0.262 \\
\hline Gender & $\begin{array}{l}\text { Male } \\
\text { Female }\end{array}$ & $\begin{array}{l}18(20.0 \%) \\
72(80.0 \%)\end{array}$ & $\begin{array}{l}9(30.0 \%) \\
21(70.0 \%)\end{array}$ & $1.290 \#$ & 0.256 \\
\hline
\end{tabular}

t-Independent Sample t-test; $\# x^{2}$ : Chi-square test; p-value >0.05 NS;

This table showed no statistically significant difference between patients and control according to demographic data.

Table (17): Comparison between patients and control according to nerve conduction study.

\begin{tabular}{|c|c|c|c|c|c|}
\hline & $\begin{array}{l}\text { Nerve conduction } \\
\text { study(Before } \\
\text { treatment) }\end{array}$ & Patients $(n=90)$ & $\begin{array}{l}\text { Control } \\
(n=30)\end{array}$ & t-test & p-value \\
\hline Distal Motor Latency & $\begin{array}{l}\text { Mean } \pm \text { SD } \\
\text { Range }\end{array}$ & \begin{tabular}{|l|}
$4.50 \pm 0.84$ \\
$2.14-6.46$ \\
\end{tabular} & $\begin{array}{l}3.51 \pm 0.26 \\
3.08-3.85 \\
\end{array}$ & 40.386 & $<0.001 * *$ \\
\hline Motor Amplitude & $\begin{array}{l}\text { Mean } \pm \text { SD } \\
\text { Range }\end{array}$ & \begin{tabular}{|l|}
$5.09 \pm 1.44$ \\
$3.11-8.56$ \\
\end{tabular} & $\begin{array}{l}7.59 \pm 0.20 \\
5.3-9.9 \\
\end{array}$ & 5.252 & $0.014 *$ \\
\hline $\begin{array}{l}\text { Motor Conduction } \\
\text { Velocity }\end{array}$ & $\begin{array}{l}\text { Mean } \pm \text { SD } \\
\text { Range }\end{array}$ & $\begin{array}{l}48.49 \pm 6.68 \\
39-67.3 \\
\end{array}$ & $\begin{array}{l}59.04 \pm 1.83 \\
46.9-68.4 \\
\end{array}$ & 6.963 & $<0.001 * *$ \\
\hline Distal Sensory Latency & $\begin{array}{l}\text { Mean } \pm \text { SD } \\
\text { Range }\end{array}$ & \begin{tabular}{|l|}
$3.92 \pm 0.98$ \\
$3.24-8.86$ \\
\end{tabular} & $\begin{array}{l}2.60 \pm 0.16 \\
2.38-2.9 \\
\end{array}$ & 53.724 & $<0.001 * *$ \\
\hline Sensory Amplitude & $\begin{array}{l}\text { Mean } \pm \text { SD } \\
\text { Range }\end{array}$ & \begin{tabular}{|l|}
$16.82 \pm 1.88$ \\
$12.5-18.7$ \\
\end{tabular} & $\begin{array}{l}25.36 \pm 1.46 \\
23.8-28 \\
\end{array}$ & 513.887 & $<0.001 * *$ \\
\hline $\begin{array}{l}\text { Sensory Conduction } \\
\text { Velocity }\end{array}$ & $\begin{array}{l}\text { Mean } \pm \text { SD } \\
\text { Range }\end{array}$ & \begin{tabular}{|l|}
$42.56 \pm 1.69$ \\
$39-47$ \\
\end{tabular} & $\begin{array}{l}48.72 \pm 1.77 \\
46.8-52.4 \\
\end{array}$ & 291.922 & $<0.001 * *$ \\
\hline
\end{tabular}


t-Independent Sample t-test, p-value >0.05 NS; **p-value <0.001 HS

This table showed statistically significant difference between patients and control according to NCS.

Table (18): Comparison between patients and control according to F-wave.

\begin{tabular}{|l|c|c|c|c|}
\hline Fwave & Patients $(\boldsymbol{n}=\mathbf{9 0})$ & Control $(\boldsymbol{n}=\mathbf{3 0})$ & t-test & p-value \\
\cline { 1 - 3 } Mean \pm SD & $26.68 \pm 1.68$ & $26.01 \pm 1.40$ & & \multirow{2}{*}{3.875} \\
\cline { 1 - 2 } Range & $23.1-29$ & $23.8-28.5$ & 0.151 \\
\hline
\end{tabular}

$t$-Independent Sample t-test; $p$-value $>0.05$ NS

This table shows no statistically significant difference between patients and control according to F-wave.

Table (19): Comparison between patients and control according to ultrasound.

\begin{tabular}{|c|c|c|c|c|c|}
\hline & Ultrasound (Before treatment) & Patients $(\mathrm{n}=90)$ & Control $(n=30)$ & $t / x 2 \#$ & p-value \\
\hline CSA & $\begin{array}{l}\text { Mean } \pm \text { SD } \\
\text { Range }\end{array}$ & $\begin{array}{c}13.69 \pm 1.25 \\
12-16.4\end{array}$ & $\begin{array}{c}9.37 \pm 0.48 \\
8.6-10\end{array}$ & 338.097 & $<0.001 * *$ \\
\hline Vascularity & $\begin{array}{l}\text { Normal } \\
\text { Minimal } \\
\text { Moderate }\end{array}$ & $\begin{array}{c}48(53.3 \%) \\
36(40.0 \%) \\
6(6.7 \%)\end{array}$ & $\begin{array}{c}30(100.0 \%) \\
0(0.0 \%) \\
0(0.0 \%)\end{array}$ & $21.538 \#$ & $<0.001 * *$ \\
\hline Mobility & Mobile & $90(100.0 \%)$ & $30(100.0 \%)$ & $0.000 \#$ & 1 \\
\hline $\begin{array}{c}\text { Flattening } \\
\text { Ratio }\end{array}$ & $\begin{array}{l}\text { Mean } \pm S D \\
\text { Range }\end{array}$ & $\begin{array}{c}3.02 \pm 0.41 \\
4-\mathrm{Feb}\end{array}$ & $\begin{array}{c}1.94 \pm 0.12 \\
2-\mathrm{Feb}\end{array}$ & 202.1 & $<0.001 * *$ \\
\hline
\end{tabular}

t-Independent Sample t-test; $\# x^{2}$ : Chi-square test; p-value >0.05 NS; **p-value <0.001 HS This table showed highly statistically significant difference between patients and control according to ultrasound.

\section{DISCUSSION}

The current study included 90 rheumatoid arthritis patients, $72(80 \%)$ females and $18(20 \%)$ males, with 62 (56\%) patients considered as mild and $28(44 \%)$ patients considered as moderate CTS. Their ages ranged from 24 to 68 years, with a mean of $46.20 \pm 12.89$ years. The rheumatoid arthritis duration ranged from 1 to 22 years, with a mean of $9.10 \pm 6.83$ years. Our study showed that local steroid injection not only provides symptomatic relief but also improves nerve conduction studies regarding both sensory and motor conduction. These results are in agreement with those obtained in the study conducted by Hagebeuk and Weerd ${ }^{(15)}$ who concluded that a single injection of steroids close to the median nerve may result in long-term improvement in CTS patients and should be considered before surgical decompression. Also, local steroid injection resulted in improvement in US measurements of the median nerve, which is in agreement with the study done by Wang $\boldsymbol{e t}$ al. ${ }^{(16)}$ who demonstrated that the US measurement of median nerve significantly improved after local steroid injection. Due to its anti-inflammatory effect, steroids are effective at reducing swelling and so considered as the cornerstone of conservative management in CTS (14). Also steroids have direct effect on the electrical properties of the myelinated nerve fibers through improvement in the functional properties and excitability of the $A \beta$ fibers, which have higher electrical threshold, after steroid injection at the wrist ${ }^{(17)}$.

In our study, the dextrose group exhibited significant reduction in VAS and improvement in nerve conduction studies regarding sensory and motor conduction and improvement of US measurement. These results are in agreement with the study done by $\mathbf{W u} \boldsymbol{e t}$ $a l .{ }^{(18)}$. This study was the first prospective, randomized, double-blind and placebo-controlled study to investigate the benefit of ultrasound guided PIT with D5W for mild to moderate CTS. A significant improvement regarding VAS and NCS was observed. Although the definite mechanism underlying the effectiveness of D5W is not clear. It is hypothesized that dextrose could decrease neurogenic inflammation by inhibiting transient receptor potential vanilloidrec eptor-1 (TRPV1). The inhibition of TRPV1 could block neurotransmitters, including calcitonin gene related peptide and substance $\mathrm{P}$, restricting neurogenic inflammation. It also stimulates the release of nerve growth factor, helping the repair and restoration of the soft tissue ${ }^{(\mathbf{1 2})}$.

Our study showed that local injection of PRP provided significant reduction in VAS and improvement in both NCS regarding both sensory and motor conduction and US measurement. These results are in agreement with Michael Alexander et al. (19) who concluded that the clinical, electrophysiological and ultrasonographic outcomes of the patients with CTS treated with PRP injections significantly improved in comparison with the baseline. It is proven that several growth factors that are released and activated after PRP injection might lead to median nerve regeneration ${ }^{(20)}$ and improve the neural blood supply by protecting the "blood-nerve barrier"(21). But, the prolotherapy group exhibited significant reduction in VAS compared to the steroid group at 3 months postinjection. This result is in accordance with Yung-Tsan et al. ${ }^{(22)}$ who concluded that there was significant reduction in pain and disability in D5W group compared to the steroid group at 3 months 
postinjection. In spite of that, NCS improvement in steroid group was greater than that in prolotherapy group. The same result has been found by Yung-Tsan et al. ${ }^{(22)}$ who detected significant improvement in NCS regarding SNCV and DML in steroid group compared with dextrose group 3 months postinjection.

As regards US measurements, there was significant reduction in CSA of the median nerve in prolotherapy group more than steroid group, although reduction in FR was more in steroid group than in prolotherapy group. These results do not go with what Yung-Tsan et al. ${ }^{\text {(22) }}$ found in their study. This discrepancy between our results and those of Yung-Tsan $\boldsymbol{e t}$ al. may be due to the difference in number of sessions of prolotherapy injection as in our study, patients received 4-6 sessions of PIT, whereas in their study, patients received only one session of PIT.

The PRP group exhibited significant reduction in VAS compared to the steroid group at 3 months postinjection. This result is in accordance with Esam $\boldsymbol{e t}$ al. ${ }^{(23)}$ who concluded that there was significant reduction in VAS in PRP group more than in steroid group at 3 months postinjection. But as regard NCS, there was significant improvement in steroid group in comparison with the PRP group. This result do not go with Esam et al. ${ }^{(23)}$ who revealed better improvement in NCS in PRP group than in steroid group and also Uzun $\boldsymbol{e t}$ al. (24) revealed no significant difference between both groups in NCS 3 months postinjection. This disagreement can be explained by selection criteria as our study contained only rheumatoid arthritis patients with a mild to moderate degree of the CTS, whereas their study included only patients with a mild to moderate degree of "idiopathic" CTS.As regards US measurements, there was significant reduction in CSA of the median nerve in steroid group more than PRP group.

Our study shows highly significant difference in NCS between patients and control whose parameters were within normal levels according to Bland ${ }^{(25)}$. As regards US measurements, there was highly significant difference between patients and control. The cross sectional area of the median nerve in patients ranged from 12 to $16.4 \mathrm{~mm}^{2}$ with mean of $13.69 \pm 1.25$ whereas the CSA of control ranged from to $10 \mathrm{~mm}^{2}$ with mean of $9.37 \pm 0.48$. These results are in accordance with Cartwright et al. ${ }^{(26)}$ who used cut offs of 8.5 to $10 \mathrm{~mm}^{2}$ for the diagnosis of CTS. The sensitivities ranged from 65 to 97 percent and the specificities ranged from 73 to 98 percent. And with Dang $\boldsymbol{e t}$ al. ${ }^{(27)}$ who stated that the normal median nerve has a cross-sectional ranging from 6.1 to 10.4 $\mathrm{mm}^{2}$, a nerve size of $12 \mathrm{~mm}^{2}$ has $99 \%$ sensitivity for diagnosing carpal tunnel syndrome. The flattening ratio of patients ranged from 2-4 with a mean of 3.02 \pm 0.41 whereas the flattening ratio of control ranged from 1.82 to 2.14 with a mean of $1.94 \pm 0.12$. These results are in partial agreement with Gyftopoulos et al. (28) who concluded that flattening ratio exceeding 4 is associated with carpal tunnel syndrome. $40 \%$ of patients had minimal power Doppler signal and 6\% had moderate power Doppler signal whereas all control were normal (no power doppler signal). These results are in agreement with Leep Hunderfund et al. ${ }^{(29)}$ who detected increased intraneural and perineural vascularity (power doppler signal) of the median nerve in CTS.

\section{CONCLUSION}

In our study we concluded that significant improvement was observed after local injection of steroid, neural prolotherapy and PRP for all the parameters measured. Thus, neural prolotherapy and PRP can be used as effective treatment choice for CTS secondary to RA. US has a complementary role in the diagnosis of peripheral nerve entrapments as US has the advantage of excellent resolution of superficial nerves and the dynamic nature of image acquisition, which makes it a natural fit for the neuromuscular and electrodiagnostic clinics. Cross sectional area (CSA) of the median nerve more than $10 \mathrm{~mm}^{2}$ and flattening ratio more than 3 is suggestive of diagnosis of carpal tunnel syndrome.

\section{REFERENCES}

1. Loveday DT, Jackson GE, Geary NP (2012): The rheumatoid foot and ankle: current evidence. Foot and Ankle Surgery, 18: 94- 102.

2 Kaeley N, Ahmad S, Pathania M et al. (2019): Prevalence and patterns of peripheral neuropathy in patients of rheumatoid arthritis. J Family Med Prim Care, 8:22-26.

3. Feldon, Paul M.D, Terrono (2006): Carpal tunnel syndrome in rheumatoid arthritis. Techniques in Orthopaedics, 21:42-47.

4. Biswas M, Ghosh S, Ghosh K et al. (2011): Prevalence, types, clinical associations, and determinants of peripheral neuropathy in rheumatoid patients. Ann Indian Acad Neural, 14:194 - 197.

5. Agarwal V, Singh R, Wiclaf et al. (2008): A clinical, electrophysiological, and pathological study

of neuropathy in rheumatoid arthritis. Clinical Rheumatol., 27:841-844.

6. Muramatsu K, Tanaka H, Taguchi T (2008): Peripheral neuropathies of the forearm and hand in rheumatoid arthritis: diagnosis and options for treatment. Rheumatol Int., 28: 951-957.

7. Ibrahim I, Medani S, El-Hameed M et al. (2013): Tarsal tunnel syndrome in patients with rheumatoid arthritis, electrophysiological and ultrasound study. Alexandria J Med., 49:95-104

8 Visser LH, Smidt MH, Lee ML (2008): High resolution sonography versus EMG in the diagnosis of carpal tunnel syndrome. J Neurol Neurosurg Psychatry, 79: 63-67.

9. Morisaki S, Nishi M, Fujiwara $\mathrm{H}$ et al. (2010): Endogenous glucocorticoids improve myelination via Schwann cells after peripheral nerve injury: an in vivo study using a crush injury model. Glia., 58(8):954-963.

10. Gooch CL, Mitten DJ (2005): Treatment of carpal tunnel syndrome: is there a role for local corticosteroid injection? Neurology, 64(12): 236- 240.

11. Dufour E, Donat N, Jaziri S et al. (2012): Ultrasound- 
guided perineural circumferential median nerveblock with and without prior dextrose 5\% hydrodissection: a prospective randomized double- blinded noninferiority trial. Anesth Analg., 115(3):728-733.

12. Wu Y, Ho T, Chou Y et al. (2017): Six-month efficacy of platelet rich plasma for carpal tunnel syndrome: a prospective randomized, single blind controlled trial. Sci Rep., 7 (1): 94.

13. Anjayani S, Wirohadidjojo YW, Adam AM et al. (2014): Sensory improvement of leprosy peripheral neuropathy in patients treated with perineural injection of plateletrich plasma. International Journal of dermatology, 53 (1): 109-113.

14. Park GY, Kwon DR (2014): Platelet-rich plasma limits the nerve injury caused by $10 \%$ dextrose in the rabbit median nerve. Muscle Nerve,49(1):56-60.

15. Hagebeuk EE, Weerd AW (2004): Clinical and electrophysiological follow- up after local steroid injection in the carpal tunnel syndrome. Clin Neurophysiol., 115(6):1464-1468.

16. Wang JC, Liao KK, Lin KP et al. (2017): Efficacy of combined ultrasound- guided steroid injection and splinting in patients with carpal tunnel syndrome: a randomized controlled trial. Arch Phys Med Rehabil., 98: 947-956

17. Cartwright MS, White DL, Demar S et al. (2011): Median nerve changes following steroid injection for carpal tunnel syndrome. Muscle Nerve, 44 (1): 25-29.

18. Bertrand H, Kyriazis M, Reeves KD et al. (2015): Topical mannitol reduces capsaicin-induced pain: results of a pilot level, double blind, randomized controlled trial. PM R., 7: 1111-1117.

19. Malahias MA, Johnson EO, Babis GC et al. (2015): Single injection of platelet-rich plasma as a novel treatment of carpal tunnel syndrome. Neural Regen Res., 10(11):18561859.

20. Sánchez M, Anitua E, Delgado D et al. (2017):
Ultrasound-guided plasma rich in growth factors injections and scaffolds hasten motor nerve functional recovery in an ovine model of nerve crush injury. J Tissue Eng Regen Med., 11 (5): 1619-29.

21. MacDermid JC, Dohert T (2004): Clinical and electrodiagnostic testing of carpal tunnel syndrome a narrative review. J Orthop Sports Phys Ther., 34(10):565588.

22. Yung-Tsan Wu, Ming-Jen Ke, Tsung-Yen Ho et al. (2018): Randomized double blinded clinical trial of 5\% dextrose versus triamcinolone injection for carpal tunnel syndrome patients. Ann Neurol., 84: 601-610.

23. Esam T, Asmaa M, Ibrahim T et al. (2018): Platelet-rich plasma versus corticosteroid injections for carpal tunnel syndrome: Clinical and electrophysiological study. https://www.sciencedirect.com/science/article/pii/S111011 6418301017

24. Uzun H, Bitik O, Uzun Ö et al. (2017): Platelet-rich plasma versus corticosteroid injections for carpal tunnel syndrome. J Plast Surg Hand Surg., 51(5):301-305.

25. Bland JD (2000): The value of the history in the diagnosis of carpal tunnel syndrome. J Hand Surg Br., 25(5):445-450.

26. Cartwright MS, Shin HW, Passmore LV et al. (2009): Ultrasonographic reference values for assessing the normal median nerve in adults. J Neuroimaging, 19(1):47-51.

27. Dang AC, Rodner CM (2009). Unusual compression neuropathies of the forearm, Part II: Median Nerve. J Hand Surg Am., 34(10):1915-1920.

28. Gyftopoulos S, Rosenberg ZS Petchprapa C (2010): Increased MR signal intensity in the pronator quadratus muscle: does it always indicate anterior interosseous neuropathy? AJR Am J Roentgenol., 194(2):490-493.

Leep Hunderfund A, Boon AJ, Mandrekar JN (2011): Sonography in carpal tunnel syndrome. Muscle Nerve, 44(4):485-491. 